\title{
The First Acanthamoeba keratitis Case of Non-Contact Lens Wearer with HIV Infection in Thailand
}

\author{
Napaporn Tananuvat', Natnaree Techajongjintana', Pradya Somboon², Anchalee Wannasan²,* \\ 'Department of Opththalmology, Faculty of Medicine, Chiang Mai University, Chiang Mai 50200, Thailand; '2Department of Parasitology, \\ Faculty of Medicine, Chiang Mai University, Chiang Mai 50200, Thailand
}

\begin{abstract}
Acanthamoeba keratitis (AK) is a rare sight-threatening corneal infection, often reporting from contact lens wearers. An asymptomatic human immunodeficiency virus (HIV)-infected Thai male without history of contact lens use complained foreign body sensation at his left eye during motorbike riding. He had neither specific keratitis symptoms nor common drugs responding, which contributed to delayed diagnosis. By corneal re-scraping, Acanthamoeba-like cysts were detected by calcofluor white staining and agar culture. The etiological agent obtained from the culture was molecularly confirmed by Acanthamoeba spp.-specific PCR, followed by DNA sequencing. The results from BLAST and phylogenetic analysis based on the DNA sequences, revealed that the pathogen was Acanthamoeba T4, the major genotype most frequently reported from clinical isolates. The infection was successfully treated with polyhexamethylene biguanide resulting in corneal scar. This appears the first reported AK case from a non-contact lens wearer with HIV infection in Thailand. Although AK is sporadic in developing countries, a role of free-living Acanthamoeba as an opportunistic pathogen should not be neglected. The report would increase awareness of AK, especially in the case presenting unspecific keratitis symptoms without clinical response to empirical antimicrobial therapy.
\end{abstract}

Key words: Acanthamoeba keratitis, non-contact lens wearer, HIV infection, calcofluor white, DNA sequencing, Thailand

\section{INTRODUCTION}

Acanthamoeba keratitis (AK) is an uncommon serious corneal infection caused by free-living protozoa in the genus Acanthamoeba. The infection routes are direct contact of corneal trauma with amoebae or exposure to contaminated dust, soil or water [1]. In developed countries, the infection frequently occurs in healthy individuals, especially in contact lens (CL) wearers with improper contact lens hygiene. However, in developing countries including Thailand, AK is a rare disease compared with other common keratitis caused by viruses, fungi and bacteria, and mostly reported from healthy hosts whose activities related to agricultural fields [1]. The incidence in developing countries is generally low, but this may be underestimation due to several reasons, such as little awareness of clinicians who are unfamiliar with the infection, or believing that AK is principally corre-

- Received 26 August 2019, revised 23 September 2019, accepted 24 September 2019. *Corresponding author (kdantra@gmail.com)

(c) 2019. Korean Society for Parasitology and Tropical Medicine

This is an Open Access article distributed under the terms of the Creative Commons Attribution Non-Commercial License (http://creativecommons.org/licenses/by-nc/4.0) which permits unrestricted non-commercial use, distribution, and reproduction in any medium, provided the original work is properly cited. lated to immunocompetent patients who wear $\mathrm{CL}$, and lack of laboratory diagnostic capacity.

Symptoms for AK are not specific, however terrible eye pain is typically disproportionate to the degree of keratitis. Clinical signs such as epithelial defects or central ulcers, anterior stromal ring infiltrates and radial keratoneuritis are commonly present [2-4]. No significant differences in signs or symptoms were detected between contact and non-contact lens related patients [2]. Diagnosis for AK relies on in vivo confocal microscopy (IVCM) or detection of amoebae from corneal scrapings by staining or agar culture of which methods need skillful observers. As symptoms of AK are nonspecific and similarly to those presenting in other microbial keratitis, delayed diagnosis or misdiagnosis may lead to prolonged therapy which cause amoebae encystment and recurrent infection. Here, we report the successful detection and treatment of AK in a non-CL wearer with HIV infection from the tertiary eye care in Northern Thailand. This study was approved by the Research and Ethics Committee, Faculty of Medicine, Chiang Mai University (study code: OPT-2561-05965) and written consent was obtained from the patient. 


\section{CASE RECORD}

A 31-year-old homosexual man with asymptomatic HIV infection had been treated at Lampang provincial hospital with severe pain, redness and photophobia of his left eye for about 3 weeks. The eye symptoms began with the foreign body sensation during motorbike riding and gradually became worse. He had neither history of HIV therapy nor experience of wearing CL. His recent CD4 count was 334 cells $/ \mathrm{mm}^{3}$. No clinical manifestation of any opportunistic infections related to HIV infection was detected. Ocular examination revealed corneal ulceration with hypopyon. A sample of corneal scraping was then taken and sent for cultures before topical antifungal and fortified antibiotics were applied. However, the scraping cul- tures were negative for both bacteria and fungus. After 6-week treatment without clinical improvement, he was referred to Chiang Mai University (CMU) hospital which is a tertiary hospital in Northern Thailand.

At CMU hospital, the visual acuity (VA) was $6 / 6$ and hand motion in the right and left eye, respectively. Slit lamp biomicroscopy of the left eye revealed dense corneal stromal infiltration involved the visual axis with overlying epithelial defect, ring infiltration and multiple satellite lesions. There was pannus formation involved the inferior half of the cornea and a layer of hypopyon in the anterior chamber (Fig. 1A, B). The intraocular pressure and the posterior eye segment evaluation by B-scan ultrasonography in the affected eye were normal. Initially, corneal scraping was performed for microbial work-
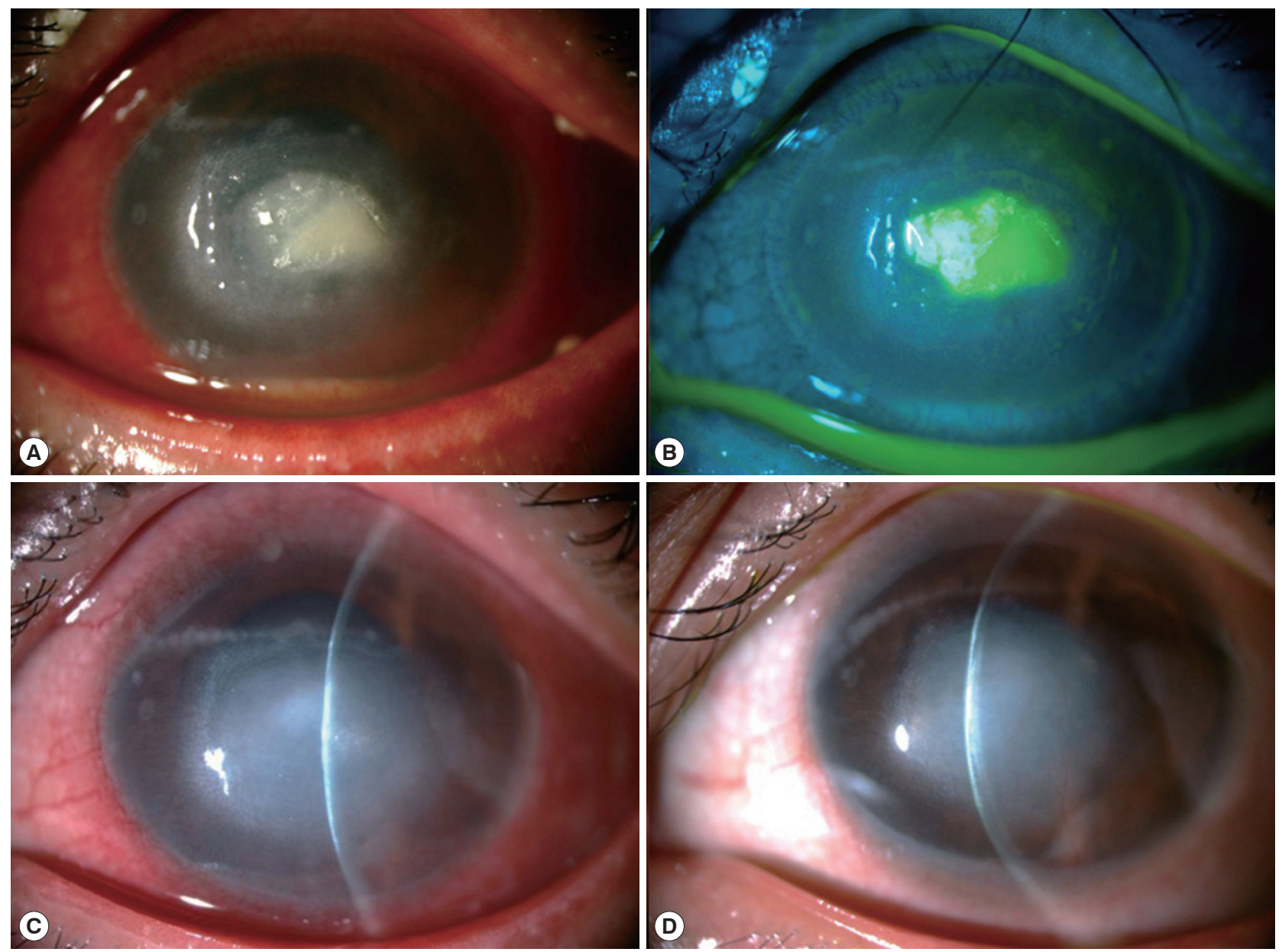

Fig. 1. Clinical photographs of the left eye. (A) Cornea had a dense stromal infiltrate involved the central part with surrounding ring infiltration, multiple satellite lesions and pannus formation involved the inferior cornea. A layer of hypopyon in the anterior chamber was present. (B) After fluorescein instillation and cobalt blue illumination, there was an epithelial defect overlaid the stromal infiltration. (C) At 2-week follow up, the stromal and ring infiltrations became resolved. (D) At 3-month follow up, the lesion turned to be a central corneal scar with minimal presence of the pannus. 
up and the specimens were subjected for microscopic exam with Gram's stain, potassium hydroxide $(\mathrm{KOH})$ wet mount, calcofluor white (CFW) stain and culture for bacteria and fungi. IVCM (Confoscan 4, Nidex Technologies, Italy) was also performed with negative results for neither fungal elements nor any microbial organisms. He was admitted and administrated with topical eye drops (5\% natamycin hourly and $0.5 \%$ levofloxacin 2 hourly and 1\% atropine 4 times/day) and oral itraconazole.

Three days after treatment, corneal conditions did not respond to the medications and the ring infiltration gradually progressed. The results of Gram's stain, $\mathrm{KOH}$ and cultures for bacteria and fungus, were all negative, while Acanthamoeba-like cysts were found by CFW staining. The cysts were distinctively fluoresced as bright blue, particularly at inner cyst wall, which clearly contrasted with corneal epithelium background (Fig. 2).

Afterward, a sample of corneal re-scraping was subjected to Acanthamoeba culture. Monoxenic culture was performed on a $1.5 \%$ non-nutrient agar plate pre-coated with heat-inactivated Escherichia coli as previously described [5]. On the second day of incubation, a clear plaque on the bacteria lawn resulting from bacterial consuming by Acanthamoeba was clearly seen. Direct observation under the light microscope, clusters of trophozoites were observed at circumferential outer rim of the plaque (Fig. 3). By wet smear preparation with saline, cysts of the isolate comprised a double-layered wall with the size less than $18 \mu \mathrm{m}$ in diameter (Fig. 4), which are unique characteristics of the cysts in Acanthamoeba group II based on Pussard and
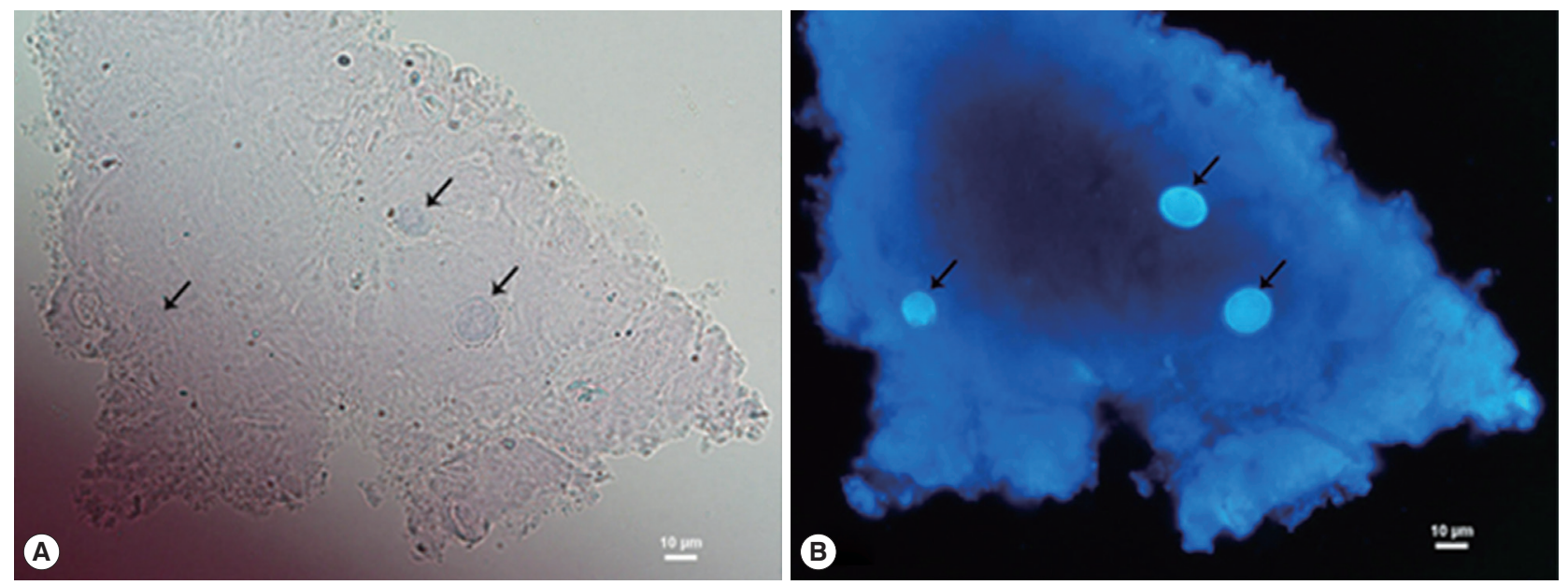

Fig. 2. Calcofluor white staining of corneal scraping showing Acanthamoeba cysts (arrows). (A) Under a bright field microscope. (B) Under a fluorescence microscope.
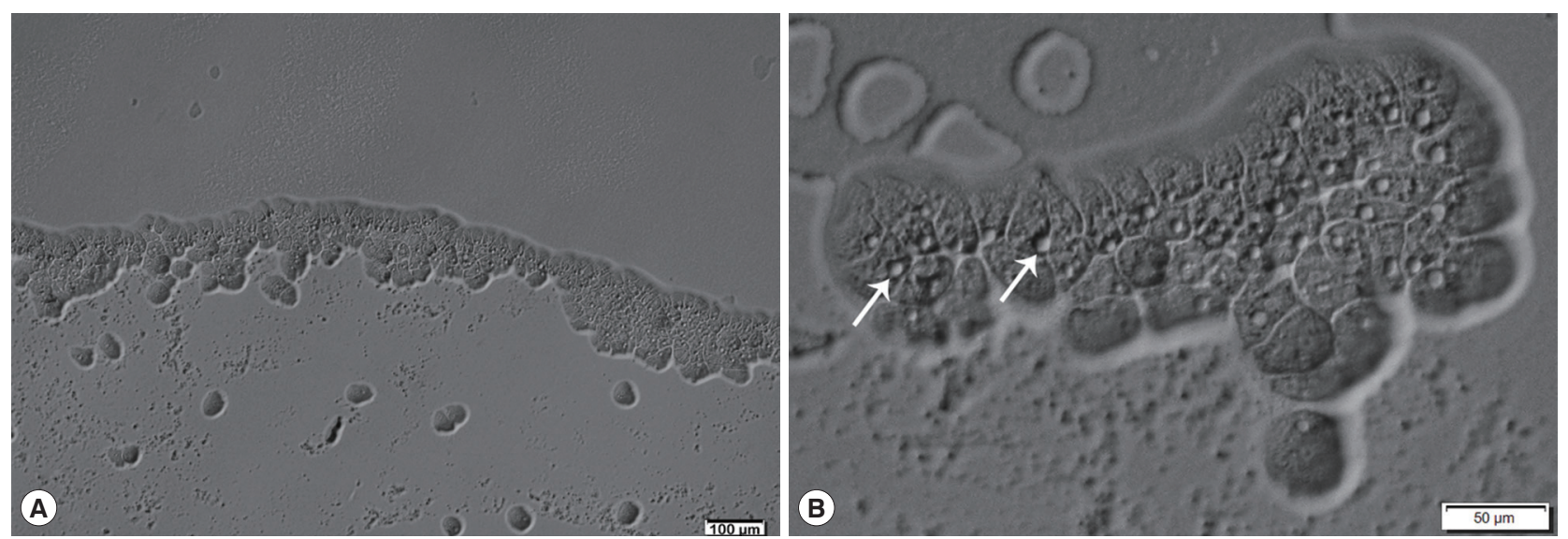

Fig. 3. Acanthamoeba trophozoites of the CMU_AK growing on monoxenic cultivation (1\% non-nutrient agar plate overlaid with E. coli). (A) Many active trophozoites locate at the border of clear zone. (B) Apparently rounded contractile vacuoles (arrows) of the trophozoites. 


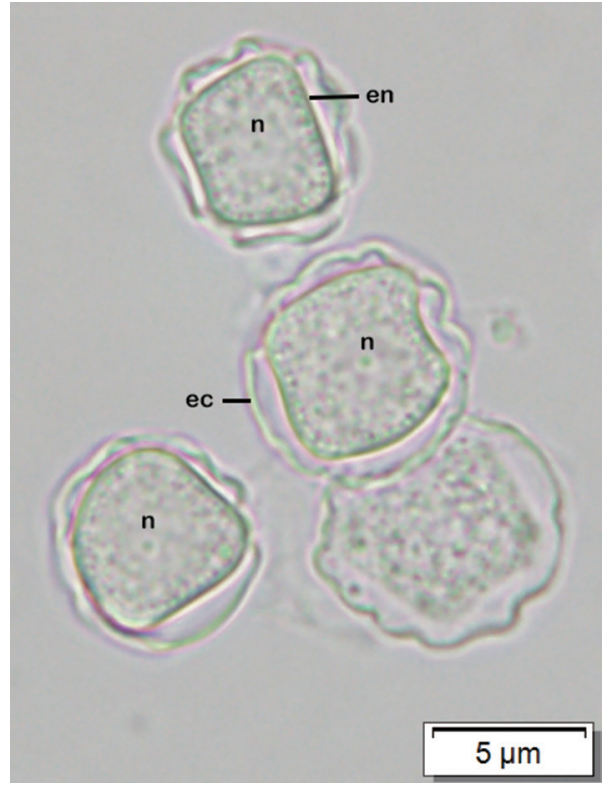

Fig. 4. Acanthamoeba cysts of the CMU_AK under the light microscope, showing nuclei (n), double-layered walls, polygonal inner walls (endocyst, en) and thick wrinkled outer walls (ectocyst, ec).

Pons' classification [6].

For molecular confirmation, genomic DNA from the Acanthamoeba isolate (called CMU_AK herein) was extracted using E.Z.N.A. ${ }^{\circledR}$ Tissue DNA Kit (Omega Biotek, Germany). The partial $18 \mathrm{~S}$ ribosomal DNA was amplified by Acanthamoeba spp.specific PCR (ACA PCR) using genus specific primers (JDP1/ JDP2) as previously described [7]. An Acanthamoeba clinical isolate from Mahidol University (MU_AK) was used as a positive control in this study. The PCR products were purified using E.Z.N.A. ${ }^{\circledR}$ Cycle Pure Kit (Omega Biotek, Doraville, Georgia, USA) and consequently sent to First BASE Laboratories Sdn. Bhd. (Selangor, Malaysia) for Sanger sequencing. The purified amplicons were bidirectionally sequenced using the same primers as previously used in the PCR. Obtained sequences were manually edited and assembled using BioEdit software version 7.0.9.0 [8]. The partial $18 \mathrm{~S}$ rDNA sequences (416 bp excluding primers) of the CMU_AK and the MU_AK were deposited in GenBank under accession numbers MH038174 and MH038175. The 2 sequences were very similar with 99.27\% pairwise identity and only 3 nucleotide differences of transition mutation were detected. For sequence similarity searches, the sequence of CMU_AK was compared with sequences in the GenBank database using the BLAST tool available at www.ncbi.nlm.nih.gov. The BLAST result revealed that Acanthamoeba sp. T4 genotype from USA (JX423600) was

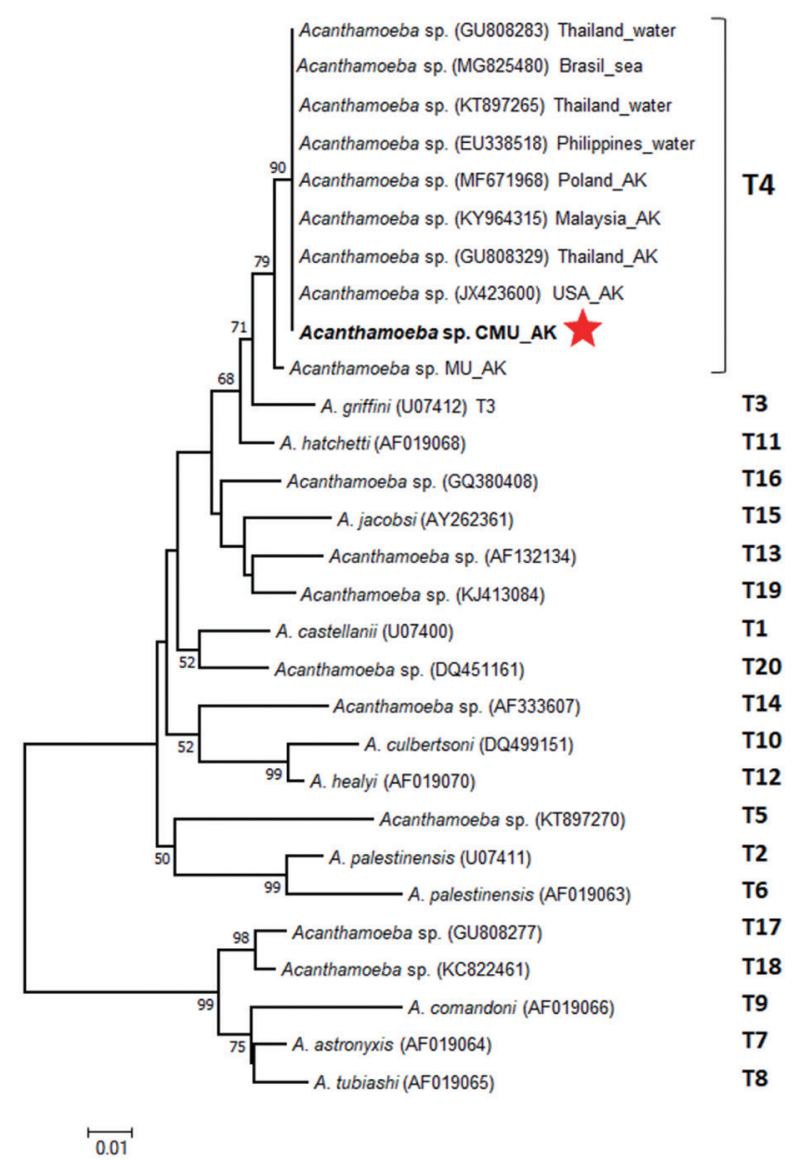

Fig. 5. Neighbor-joining tree based on partial 18S rDNA sequences of Acanthamoeba spp. (genotype T1-T20). A star indicated the position of the CMU_AK. Accession numbers of the references were provided in parentheses. Bootstrap values (>50\%) are shown near appropriate nodes. A scale bar 0.01 indicates the evolutionary distance divergence.

the first sequence hit to that of the CMU_AK with 100\% identity.

To classify Acanthamoeba genotype, neighbor-joining (NJ) phylogenetic tree based on the partial 18S rDNA sequence alignment of the CMU_AK, the MU_AK and 20 representative genotypes (T1-T20) accessible from the GenBank, was conducted through MEGA6 software [9] using the Kimura 2-parameter method [10] with 1,000 bootstrap replications. The tree topology presented 2 main distinct clades associated with the cyst morphology categorized by Pussard and Pons [6] (Fig. 5). The big clade comprised of the CMU_AK, the MU_AK and other Acanthamoeba references corresponding to Acanthamoeba group II or group III (cyst diameter $<18 \mu \mathrm{m}$ ), while the small clade consisted of Acanthamoeba group I (cyst diameter $>18$ $\mu \mathrm{m})[6,11]$. Analyzed genotypes were clearly separated into 
their own monophyletic clade. The CMU_AK was clustered into T4 clade together with the MU_AK and other Acanthamoeba T4 references (Fig. 5).

After the causative agent was clarified, the patient was then treated with topical polyhexamethylene biguanide (PHMB) hourly. On the third day after treatment, the ring infiltration was resolved, therefore the frequency of PHMB application was gradually reduced to 2 hourly and the patient was discharged home after a week of the therapy.

At 2-week follow-up, the stromal infiltration resolved with small epithelial defect and mild anterior chamber reaction (Fig. 1C). Topical PHMB was tapered down to every $4 \mathrm{hr}$. At 3-month follow-up, the infiltration became central scar with corneal pannus (Fig. 1D). The pin hole-corrected VA was improved to $6 / 18$ in the affected eye.

\section{DISCUSSION}

Unlike systemic Acanthamoeba infection that mostly associated with immunocompromised hosts, AK was generally reported from immunocompetent hosts, especially in CL wearer. The improper usage of $\mathrm{CL}$ is believed to be a primarily risk factor of AK outbreak in developed countries such as USA [12], England [13], Hong Kong [14], and Singapore [15]. However, incidence of AK in developing countries is often related with outdoor activities and risk exposures to dust, soil or water [1]. In Thailand, Acanthamoeba have been isolated from clinical and environmental samples [5,16-19]. Despite its ubiquity in the nature, less than $10 \mathrm{AK}$ cases have been previously reported from Thailand [20], bringing up to the curiosity that the true AK incidence may be underestimated.

This study appears to be the first AK case in Thailand occurred in a HIV-infected patient without history of CL wearing. The patient presented atypical ocular findings and was initially misdiagnosed as fungal keratitis. Two months after the onset, delayed diagnosis caused permanent corneal scar and visual loss. The longer diagnostic period and the poorer visual outcome have also been reported in non-CL-related AK series, which are the significant points differed from CL-related AK [2]. In immunosuppressed patients, Acanthamoeba can cause granulomatous encephalitis and disseminated acanthamoebiasis [21]. As an opportunistic role, Acanthamoeba can cause spontaneous keratitis in HIV-infected patients [22] or even polymicrobial infection [23]. The risk of Acanthamoeba keratitis in immunocompromised host has neither been increased than normal host nor associated with a systemically immunocompromised state [24]. The lack of typical signs of Acanthamoeba keratitis, particularly in non-contact lens wearer, can lead to the delay in diagnosis and unsatisfactory outcomes or even loss of the eye [22]. However, the relationship between HIV infection and Acanthamoeba keratitis remained unknown.

For diagnosis, the causative agent in this study was eventually approved by CFW staining, a non-specific fluorochrome extensively used in clinical mycology. Principally, CFW is composed of compounds which has the binding ability to chitin and cellulose on the microorganism's structures [25]. In parasitological field, CFW is known as an optical fluorescence brightener widely used in the rapid detection for spores of microsporidia [26], cysts of Entamoeba [27], cysts and trophozoites of Giardia lamblia [28], cysts of Pneumocystis carinii [29], eggs of Ascaris lumbricoides [30], larvae of Dirofilaria immitis [31] and cysts of Acanthamoeba [32]. In this study, Acanthamoeba in the specimen appeared mainly the cyst form due to the prolonged infection without therapeutic responses. The endocysts features which made up mainly of cellulose might enhance the detection possibility by CFW. The bright bluish-white fluorescence furnished the endocysts so that they could be easily distinguished from surrounding corneal tissue background. Our results were concordant with the previous investigation and insisted the robustness of CFW staining in AK diagnosis that CFW staining of repeated corneal scrapings was helpful, reliable enough to be the decisive method, especially in nonspecific symptomatic cases [33].

The routine tests for $\mathrm{AK}$ diagnosis in most laboratories are generally microscopy of corneal scraping and culture. By comparison with smear staining, culture method may take longer detection time which may be up to 2 weeks in some cases depending on quantity of amoebae available in the samples, and the quality of corneal scrapings. Fortunately, in our case, amoebae amount in the scraping was rich enough to produce the clear zone on the agar culture within the second day of incubation. In some cases that microscopic exam showed negative, the culture which simultaneously performed may also be helpful [34]. Moreover, the clinical isolated amoebae harvested from the culture can be used for further epidemiological or genetic studies.

For molecular diagnosis, Acanthamoeba spp.-specific PCR (ACA-PCR) has been recently developed revealing high sensitivity and specificity in the rapid AK detection $[7,35]$. It was noted from an AK study in non-CL Indian wearer that sensitiv- 
ity of the PCR would have been higher if the samples were from initial corneal scraping rather than the latter [34]. As Acanthamoeba-specific amplimer S1 (so called ASA.S1) retrieved from the ACA-PCR shows high variability among individual genotypes, the PCR has been extensively used for both clinical and epidemiological objectives. Based on the variations in $18 \mathrm{~S}$ rDNA sequences, genus Acanthamoeba can currently be classified into 20 diverse genotypes (T1-T20) [11]. Among them, T4 is a major genotype commonly isolated from patients and nature. In the present study, the causative agent CMU_AK was designated as typical T4 genotype which was concordantly affirmed by BLAST and phylogenetic analysis. The sequence of CMU_AK was identical to that of all Acanthamoeba $\mathrm{T} 4$ references from keratitis patients and environments but showed $0.73 \%$ nucleotide differences from that of the positive control, MU_AK. The clustering of the MU_AK into T4 monophyletic clade, could be explained that the $0.73 \%$ of genetic distances were remained lower than the $5 \%$ cut off value which is known to be characteristic of different genotype [11]. To our knowledge, few molecular studies of Acanthamoeba isolated in Thailand have been documented $[18,19,36,37]$. Clinically, genotypes T4 and T10 were reported to be responsible for Acanthamoeba keratitis cases, while only T4 was identified from a case of Acanthamoeba meningoencephalitis [18]. Environmentally, genotypes T3, T4, T5, T9, and T17 were isolated from natural water resources of Thailand $[18,19]$. It is likely that $\mathrm{T} 4$ is the most widespread distribution genotype in the country. Although not all T4 isolates have been reported to possess pathogenic properties, majority of Acanthamoeba infections have been associated with genotype $\mathrm{T} 4$ [21]. Therefore, potential pathogenicity of T4 reporting in the country should not be ignored. The disease monitoring in human groups involving the risk activities like agriculture or joining of water-related festivals such as Loi Krathong and Songkran, should be aware.

In conclusions, the clinical manifestations of Acanthamoeba keratitis in HIV-infected patient may be atypical making the diagnosis is delayed and resulting in poor visual outcome. Comprehensive history taking and combination of diagnostic modalities are important to prevent the misdiagnosis. Although Acanthamoeba can play an opportunistic role in HIVinfected patient with suboptimal immune status, it is still unclear whether this influenced the course of the disease.

\section{ACKNOWLEDGMENTS}

We would like to thank Asst. Prof. Kosol Roongruangchai for providing an Acanthamoeba isolate used as positive control in this study. This study was supported by a research grant of the Bio \& Medical Technology Development Program of the National Research Foundation (NRF) funded by the Ministry of Science \& ICT, South Korea (grant number: 2017M3A9E40707).

\section{CONFLICT OF INTEREST}

We have no conflict of interest related to this work.

\section{REFERENCES}

1. Ibrahim YW, Boase DL, Cree IA. Factors affecting the epidemiology of Acanthamoeba keratitis. Ophthalmic Epidemiol 2007; 14: 53-60.

2. Chynn EW, Lopez MA, Pavan-Langston D, Talamo JH. Acanthamoeba keratitis. Contact lens and noncontact lens characteristics. Ophthalmology 1995; 102: 1369-1373.

3. Moore MB, McCulley JP, Kaufman HE, Robin JB. Radial keratoneuritis as a presenting sign in Acanthamoeba keratitis. Ophthalmology 1986; 93: 1310-1315.

4. Theodore FH, Jakobiec FA, Juechter KB, Ma P, Troutman RC, Pang PM, Iwamoto T. The diagnostic value of a ring infiltrate in acanthamoebic keratitis. Ophthalmology 1985; 92: 1471-1479.

5. Wannasan A, Uparanukraw P, Songsangchun A, Morakote N. Potentially pathogenic free-living amoebae in some flood-affected areas during 2011 Chiang Mai flood. Rev Inst Med Trop Sao Paulo 2013; 55: 411-416.

6. Pussard M. Morphologie de la paroi kystique et taxonomie du genre Acanthamoeba (Protozoa, Amoebida). Protistologica 1977; 13: $557-598$

7. Schroeder JM, Booton GC, Hay J, Niszl IA, Seal DV, Markus MB, Fuerst PA, Byers TJ. Use of subgenic $18 \mathrm{~S}$ ribosomal DNA PCR and sequencing for genus and genotype identification of acanthamoebae from humans with keratitis and from sewage sludge. J Clin Microbiol 2001; 39: 1903-1911.

8. Hall TA. Bioedit: a user-friendly biological sequence alignment editor and analysis program for Windows 95/98/NT. Nucleic Acids Symp Ser 1999; 41: 95-98.

9. Saitou N, Nei M. The neighbor-joining method: a new method for reconstructing phylogenetic trees. Mol Biol Evol 1987; 4: 406-425.

10. Kimura M. A simple method for estimating evolutionary rate of base substitutions through comparative studies of nucleotide sequences. J Mol Evol 1980; 16: 111-120.

11. Fuerst PA, Booton GC, Crary M. Phylogenetic analysis and the evolution of the 18S rRNA gene typing system of Acanthamoeba. 
J Eukaryot Microbiol 2015; 62: 69-84

12. Verani JR, Lorick SA, Yoder JS, Beach MJ, Braden CR, Roberts JM, Conover CS, Chen S, McConnell KA, Chang DC, Park BJ, Jones DB, Visvesvara GS, Roy SL. Acanthamoeba keratitis Investigation Team. National outbreak of Acanthamoeba keratitis associated with use of a contact lens solution. United States. Emerg Infect Dis 2009; 15: 1236-1242.

13. Carnt N, Hoffman JJ MBBS, Verma S, Hau S, Radford CF, Minassian DC, Dart JKG. Acanthamoeba keratitis: confirmation of the UK outbreak and a prospective case-control study identifying contributing risk factors. Br J Ophthalmol 2018; 102: 1621-1628.

14. Lam DS, Houang E, Fan DS, Lyon D, Seal D, Wong E. Incidence and risk factors for microbial keratitis in Hong Kong: comparison with Europe and North America. Eye (Lond) 2002; 16: 608618.

15. Por YM, Mehta JS, Chua JL, Koh TH, Khor WB, Fong AC, Lim JW, Heng WJ, Loh RS, Lim L, Tan DT. Acanthamoeba keratitis associated with contact lens wear in Singapore. Am J Ophthalmol 2009; 148: 7-12 e12.

16. Nacapunchai D, Kino H, Ruangsitticha C, Sriwichai P, Ishih A, Terada M. A brief survey of free-living amebae in Thailand and Hamamatsu District, Japan. Southeast Asian J Trop Med Public Health 2001; 32 (suppl): 179-182.

17. Lekkla A, Sutthikornchai C, Bovornkitti S, Sukthana Y. Free-living ameba contamination in natural hot springs in Thailand. Southeast Asian J Trop Med Public Health 2005; 36 (suppl): 5-9.

18. Nuprasert W, Putaporntip C, Pariyakanok L, Jongwutiwes S. Identification of a novel 17 genotype of Acanthamoeba from environmental isolates and $\mathrm{t} 10$ genotype causing keratitis in Thailand. J Clin Microbiol 2010; 48: 4636-4640.

19. Thammaratana T, Laummaunwai P, Boonmars T. Isolation and identification of Acanthamoeba species from natural water sources in the northeastern part of Thailand. Parasitol Res 2016; 115: 1705-1709.

20. Wannasan A. Acanthamoeba. Chiang Mai, Thailand. Jarus Business Printing. 2018, pp 69-73 (In Thailand).

21. Khan NA. Acanthamoeba: Biology and Pathogenesis. Norfolk, UK. Caister Academic Press. 2009, pp 89-90.

22. Hansen B, Kronborg G. Acanthamoeba keratitis in a non-contact lens wearer with human immunodeficiency virus. Scand J Infect Dis 2003; 35: 207-209.

23. Tandon R, Vajpayee R, Gupta V, Vajpayee M, Satpathy G, Dada T. Polymicrobial keratitis in an HIV-positive patient. Indian J Ophthalmol 2003; 51: 87-88.

24. Illingworth CD, Cook SD. Acanthamoeba keratitis. Surv Ophthal- mol 1998; 42: 493-508.

25. Harrington BJ, Hageage GJ. Calcofluor white: a review of its uses and applications in clinical mycology and parasitology. Lab Med 2003; 34: 361-367.

26. Monheit JG, Brown G, Kott MM, Schmidt WA, Moore DG. Calcofluor white detection of fungi in cytopathology. Am J Clin Pathol 1986; 85: 222-225.

27. Arroyo-Begovich A, Carabez-Trejo A, Ruiz-Herrera J. Identification of the structural component in the cyst wall of Entamoeba invadens. J Parasitol 1980; 66: 735-741.

28. Ward HD, Alroy J, Lev BI, Keusch GT, Pereira ME. Identification of chitin as a structural component of Giardia cysts. Infect Immun 1985; 49: 629-634.

29. Fraire AE, Kemp B, Greenberg SD, Kim HS, Estrada R, McBride RA. Calcofluor white stain for the detection of Pneumocystis carinii in transbronchial lung biopsy specimens: a study of 68 cases. Mod Pathol 1996; 9: 861-864.

30. Green LK, Cowan DF, Moore DG. Examination of tissue for parasites by fluorescent microscopy. Histopathol 1988; 12: 553555.

31. Green LK, Ansari MQ, Schwartz MR, Ro JY, Alpert LC. Non-specific fluorescent whitener stains in the rapid recognition of pulmonary dirofilariasis: a report of 20 cases. Thorax 1994; 49: 590593.

32. El-Sayed NM, Hikal WM. Several staining techniques to enhance the visibility of Acanthamoeba cysts. Parasitol Res 2015; 114: 823830.

33. Jain R, Garg P, Motukupally SR, Geary MB. Clinico-microbiological review of non-contact-lens-associated Acanthamoeba keratitis. Semin Ophthalmol 2015; 30: 281-288.

34. Pasricha G, Sharma S, Garg P, Aggarwal RK. Use of 18 S rRNA gene-based PCR assay for diagnosis of Acanthamoeba keratitis in non-contact lens wearers in India. J Clin Microbiol 2003; 41: 3206-3211.

35. Boggild AK, Martin DS, Lee TY, Yu B, Low DE. Laboratory diagnosis of amoebic keratitis: comparison of four diagnostic methods for different types of clinical specimens. J Clin Microbiol 2009; 47: 1314-1318.

36. Jongwutiwes S, Pariyakanok L, Charoenkorn M, Yagita K, Endo T. Heterogeneity in cyst morphology within isolates of Acanthamoeba from keratitis patients in Thailand. Trop Med Int Health 2000; 5: 335-340.

37. Wara-Asawapati S, Intapan PM, Chotmongkol V. Acanthamoeba brain abscess confirmed by molecular identification. Am J Trop Med Hyg 2017; 97: 307-308. 
